\title{
DESIGN OF UEP-BASED MSE-MINIMIZING RATELESS CODES FOR SOURCE-CHANNEL CODING
}

\author{
Amirpasha Shirazinia, Lei Bao, Mikael Skoglund \\ Royal Institute of Technology, Stockholm, Sweden \\ Email: \{amishi, lei.bao, skoglund\}@ee.kth.se
}

\begin{abstract}
This paper proposes a method to optimize the performance of tandem source-channel coding with respect to the mean-squared error by exploiting the unequal error protection coding. More specifically, we formulate a combination of linear programming and grid search to optimize degree distributions for unequal error protected rateless channel codes. An asymptotic upper bound for the meansquared error of the cascaded system is also derived. By optimizing the corresponding degree distributions of the rateless codes using unequal error protection principles, the proposed scheme has shown promising performance at high resolution region of source coding.
\end{abstract}

Index Terms- Source-channel coding, rateless codes, unequal error protection, degree distribution, mean-square error

\section{INTRODUCTION}

Conventionally, source coders are introduced for transferring source information with fewer bits or representing analog sources. Channel coders are designed to protect data transmission over noisy channels. Although the optimization of source and channel coders can be performed jointly, the codes are often designed separately because of practical reasons. The source-channel separation theorem states that under certain conditions, such as delay constraint, combining separately designed source and channel codes still achieves the optimal performance for transmission over noisy channels [1]. On the other hand, a joint design is useful when delay constraint is strict. However, the complexity of joint optimization becomes significantly expensive for systems with large information to be transmitted. To compensate the delay in separate source-channel coding, one might consider priority for sensitive source information bits and transmit them more frequently. Therefore, by adding some amount of coupling between source and channel coders using unequal error protection (UEP) the performance can be maintained in noisy environment by means of protecting more significant information under a given delay constraint. This is a subject of anytime coding in [2], where the nature of information bits changes from equally-error protected (EEP) into the one with different priorities to be treated in order for the quality of results to gradually improve as computation time increases. In [3], the authors used UEP principles for source-channel coding as well. Rateless codes are a class of channel codes in which the encoder produces an unlimited number of symbols such that the decoder can recover the source symbols from a sufficiently large subset of channel outputs. Exploiting rateless codes for UEP applications have been studied in $[4,5]$, where the authors showed that using this aspect, sensitive bits can be effectively protected in applications such as scalable video multi-cast. Luby Transform (LT) codes [6] are rateless error control codes which are suitable schemes for erasure channels. The results in [7] also show that LT and Raptor codes[8] (concate- nation of LT codes with high-rate LDPC outer encoder/decoder) perform very well on symmetric noisy channels such as binary symmetric channels (BSC) and binary-input additive white Gaussian noise (BIAWGN) channels.

In this paper, we propose a joint design of cascaded sourcechannel codes based on UEP property. In particular, we study the performance of cascaded high-resolution quantizer and rateless channel coder with the aim of optimizing degree distributions of UEP-LT codes. We also provide an asymptotic upper bound for end-to-end mean-squared error (MSE) of the proposed system at high resolution source coding. To the best of our knowledge, the problem of optimizing degree distribution for UEP-LT codes with the objective of minimizing MSE has not been considered before. Therefore, in this paper, we focus on optimizing degree distributions of UEP-LT codes, and evaluating the performance of source-channel coding system using the optimized UEP-LT codes. We will also compare the performance of the proposed design with unoptimized UEP-LT codes in order to quantify the gain achieved by optimizing the degree distributions.

\section{PROBLEM STATEMENT}

We consider a non-negative real random variable $X$ which is first quantized using a truncated dyadic expansion as $X_{Q}=\sum_{k=1}^{K} \lambda^{-k} b_{k}$, where $b_{k} \in\{0,1\}$ are independent and identically distributed (iid) random variables, $\lambda>1$, and $K$ represents the number of information bits. Then, the bits $b_{k}$ are encoded using a channel-coding technique and transmitted over a binary symmetric channel with bit cross-over probability $\epsilon$. The estimation of bits at the decoder $\hat{b}_{k}$ is then followed by an inverse binary expansion which results in the reconstructed value $\hat{X}$. We define source distortion $\mathrm{MSE}_{Q}$, channel distortion $\mathrm{MSE}_{c h}$, and ene-to-end distortion $\mathrm{MSE}_{e 2 e}$ as

$$
\begin{aligned}
& \mathrm{MSE}_{Q} \triangleq \mathbb{E}\left[\left(X-X_{Q}\right)^{2}\right], \mathrm{MSE}_{c h} \triangleq \mathbb{E}\left[\left(X_{Q}-\hat{X}\right)^{2}\right], \\
& \mathrm{MSE}_{e 2 e} \triangleq \mathbb{E}\left[(X-\hat{X})^{2}\right] .
\end{aligned}
$$

The distortion measures in (1) are related as

$$
\begin{aligned}
\operatorname{MSE}_{e 2 e} & =\mathbb{E}\left[\left(X-X_{Q}+X_{Q}-\hat{X}\right)^{2}\right] \\
& \stackrel{(a)}{\leq}\left(\mathbb{E}^{1 / 2}\left[\left(X-X_{Q}\right)^{2}\right]+\mathbb{E}^{1 / 2}\left[\left(X_{Q}-\hat{X}\right)^{2}\right]\right)^{2} \\
& =\left(\mathrm{MSE}_{Q}^{1 / 2}+\mathrm{MSE}_{c h}^{1 / 2}\right)^{2},
\end{aligned}
$$

where $(a)$ follows from Minkowski inequality. In (2), the source distortion $\mathrm{MSE}_{Q}$ tends to zero as $K$ increases, therefore, $\mathrm{MSE}_{e 2 e}=$ $\mathrm{MSE}_{c h}$, so we focus on $\mathrm{MSE}_{c h}$. In this paper, we assume that $X$ 
is uniformly distributed over a unitary interval, therefore,

$$
\begin{aligned}
\mathrm{MSE}_{c h} & =\sum_{k=1}^{K} \lambda^{-2 k} \mathbb{E}\left[\left(b_{k}-\hat{b}_{k}\right)^{2}\right] \\
& \stackrel{(a)}{=} \sum_{k=1}^{K} \lambda^{-2 k} \operatorname{Pr}\left(\hat{b}_{k}=1 \mid b_{k}=0\right) \stackrel{(b)}{=} \sum_{k=1}^{K} \lambda^{-2 k} P_{e}(k),
\end{aligned}
$$

where $(a)$ follows from the fact that input bits are independent and equi-probable, and $(b)$ follows by defining $\operatorname{Pr}\left(\hat{b}_{k}=1 \mid b_{k}=\right.$ $0) \triangleq P_{e}(k)$. As a special case, assume that no channel codes are used, then the MSE given by (3) is equivalent to $\epsilon \sum_{k=1}^{K} \lambda^{-2 k}$ which implies that the MSE increases linearly with the channel bit error probability. Throughout the paper, the measure of performance is $\mathrm{MSE}_{e 2 e}$ which is equivalent to channel distortion at high-resolution region of source coding, i.e., at large $K$. It is important to establish a relation between the source and channel coders such that they jointly decrease the overall MSE. In fact, if the probability of error, $P_{e}(k)$, in (3) is different for bits in such a way that the more significant ones have less error using UEP principles, then the performance can be significantly improved. On the other hand, the number of bits to be transmitted is large because of high resolution of quantization region. Therefore, rateless codes can be suitable channel codes since the error probability becomes very low by adding a small parity overhead. The motivation is now to design rateless codes based on optimized degree distributions by taking the UEP property for source channel coding into account so as to minimize $\mathrm{MSE}_{e 2 e}$.

\section{UEP-LT CODE DESIGN}

In this section, we first describe the encoding and decoding algorithms for LT codes. Thereafter, in Section 3.2 an asymptotic (with respect to block length) upper bound of the performance is derived by taking the UEP property into account. Later, in Section 3.3 the optimization problem for degree distributions of the UEP-LT codes used in the cascaded source-channel coding system will be formulated and solved.

\subsection{An Overview of LT Codes}

LT codes are shown to achieve the Shannon limit for channels with erasures. The encoder of LT codes generates an endless stream of bits in such a way that each codeword is produced by XOR'ing of uniformly randomly chosen input bits based on a degree distribution. We follow the notations in [8] to introduce the design parameters of LT codes. Let $\Omega(x)=\sum_{d=1}^{D} \Omega_{d} x^{d}$ denote the output node degree distribution, where $\Omega_{d}$ represents the probability of choosing corresponding number of input bits. We define output edge degree distribution as $\omega(x)=\sum_{d} \omega_{d} x^{d-1}=\Omega^{\prime}(x) / \Omega^{\prime}(1)$, and also average degree of an output bit as $\beta=\Omega^{\prime}(1)$. Similar degree distributions can be specified for input bits. Let $I(x)=\sum_{i} I_{i} x^{i}$ denote the input node degree distribution, where $I_{i}$ is the probability that the input node is the neighbor of $n(n=1, \ldots, N)$ output nodes, and let $\iota(x)=\sum_{i} \iota_{i} x^{i-1}=I^{\prime}(x) / I^{\prime}(1)$ represent the input edge degree distribution. In addition, $\alpha=I^{\prime}(1)$ is defined as average degree of an input bit. In order to calculate $I_{i}$, we fix an input bit, the probability that it is a neighbor of an output bit is $\beta / K$. After receiving $N$ output bits, the probability that an input bit is of degree $i$ is $\left(\begin{array}{c}N \\ i\end{array}\right)(\beta / K)^{i}(1-\beta / K)^{N-i}$; thus, $I(x)=\sum_{i}\left(\begin{array}{c}N \\ i\end{array}\right)(\beta / K)^{i} x^{i}(1-\beta / K)^{N-i}$. Note that $\beta / K=\alpha / N$.

At the receiver, belief propagation (BP) algorithm has been regarded as one of the successful algorithms in decoding graphical

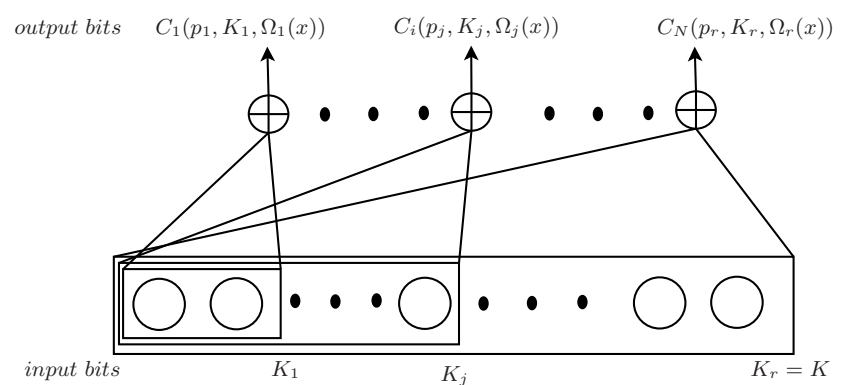

Figure 1: UEP-LT encoding scheme.

codes approaching Shannon capacity. It is an iterative algorithm that at each iteration input and output nodes exchange messages, containing log-likelihood ratio (LLR). We follow the notation of [7] for updating rules of the messages passed at iteration $l \geq 0$. Let $m_{i, o}^{(l)}\left(m_{o, i}^{(l)}\right)$ denote the messages passed from an input (output) to an output (input) node at the $l^{\text {th }}$ iteration of BP, then

$\tanh \left(m_{o, i}^{(l)} / 2\right)=\tanh (z / 2) \prod_{i^{\prime} \neq i} \tanh \left(m_{i^{\prime}, o}^{(l)} / 2\right), m_{i, o}^{(l+1)}=\sum_{o^{\prime} \neq o} m_{o^{\prime}, i}^{(l)}$

where $z \triangleq \ln [\operatorname{Pr}(x=0) \mid Y) / \operatorname{Pr}(x=1) \mid Y)]$ denotes channel LLR, and $Y$ is the channel output. At the last iteration, the LLR of the input bit $i, \sum_{o} m_{o, i}^{(l)}$, is a measure to decide if the decoded bit is 1 or 0 , where the sum is over all output bits adjacent to the input bit.

In order to analyze the performance of the BP algorithm, density evolution (DE) [9] is considered to be a standard approach for analyzing graphical codes where the block length is very large. For simplicity, instead of tracking the densities in DE, one can approximate the probability density of messages passed at each iteration as a particular form. One common density used in several literatures (e.g., $[7,10])$ is Gaussian in which $m_{i, o}$ and $m_{o, i}$ are approximated by Gaussian variables with mean and variance which can be tracked. We will assume Gaussian approximation in order to asymptotically analyze the performance in the next Section.

\subsection{Asymptotic Analysis of MSE for UEP-LT Codes}

In the framework introduced in Section 2, it is important to characterize the source coded bits to protect the important ones more securely. In the simplest case, the bits can be separated into most important bits (MIB) and least important bits (LIB). This strategy can be performed for LT codes in the following technique which is called expanding window Fountain (EWF) codes[5]. For simplicity, we demonstrate the concept using two windows where the first one contains $K_{1}$ bits, and the second window contains $K_{2}=K$ bits. There is also a probability $p$ that specifies which window is selected, i.e., with probability $p$ (or, $1-p$ ), the bits in the first window (or, the second window) are chosen to be XOR'ed and construct the codewords based on a degree distribution $\Omega_{1}(x)$ (or, $\Omega_{2}(x)$ ). This method can be simply extended to $r$-level UEP-LT codes $(2<r \leq K)$ with $r$ levels of protection. After receiving $N$ output bits, it is easy to show that the input bits in the $j^{\text {th }}$ window generates $N_{j}=p_{j} N$ output bits. Moreover, this method produces $r$ levels of importance where the $j^{t h}$ one contains $K_{j}-K_{j-1}$ bits. Therefore, the windows' length $K_{j}$, the window selection probability $p_{j}$, and the degree distributions $\Omega_{j}(x)=\sum_{d=1}^{D_{j}} \Omega_{j, d} x^{d}$ $(j=1, \ldots, r)$ are the design parameters. Figure 1 shows the encoding scheme. If we consider the $r$-level UEP-LT scheme and run the 
BP algorithm for $l$ iterations, by combining (2) and (3) we obtain

$$
\operatorname{MSE}_{e 2 e}^{(l)}=\sum_{j=1}^{r} \sum_{k_{j}=1+K_{j-1}}^{K_{j}} \lambda^{-2 k_{j}} P_{e_{j}}^{(l)},
$$

where $P_{e_{j}}^{(l)}$, assuming all-zero codeword is sent, is calculated as

$$
P_{e_{j}}^{(l)}=\operatorname{Pr}\left\{\sum_{o} m_{o, i}^{j,(l)}<0\right\}=\sum_{n} I_{n, j} Q\left(\sqrt{n \mathbb{E}\left[m_{o, i}^{j,(l)}\right] / 2}\right),
$$

where $Q(\cdot)$ is the Q-function, and we used the fact that the messages passed at the $l^{\text {th }}$ iteration of the BP from output to input nodes are symmetrically Normally distributed and have the same densities, i.e., the message from an output bit to an input bit of the $j^{t h}$ window $m_{o, i}^{j,(l)} \sim \mathcal{N}\left(\mathbb{E}\left[m_{o, i}^{j,(l)}\right], 2 \mathbb{E}\left[m_{o, i}^{j,(l)}\right]\right)$. Furthermore, $I_{n, j}$ denotes the probability that a randomly chosen bit in the $j^{\text {th }}$ level of importance is of degree $n$. Applying the asymptotic approximation $I_{j}(x) \approx \exp \left(\alpha_{j}(x-1)\right)$ [7] and the bound $Q(x) \leq \frac{1}{2} \exp \left(-x^{2} / 2\right)$ to $(5)$, yields

$\mathrm{MSE}_{e 2 e}^{(l)} \leq \frac{1}{2} \sum_{j=1 k_{j}}^{r} \lambda^{-2 k_{j}} \exp \left\{\alpha_{j}\left(\exp \left(-\frac{\mathbb{E}\left[m_{o, i}^{j,(l)}\right]}{4}\right)-1\right)\right\}$,

where $\alpha_{j}=\sum_{i=j}^{r} p_{i} \beta_{i} N / K_{i}$ and $\beta_{j}=\Omega_{j}^{\prime}(1)$ denote the average degree of an input bit and an output bit in the $j^{\text {th }}$ level of importance and the $j^{t h}$ window, respectively. Since the message densities are assumed to be the same, the updating rule for $\mathbb{E}\left[m_{o, i}^{j,(l)}\right]$ can be determined by considering the messages passed from the output bits to the input bits of the first window only. The updating rule for original LT codes [7] can be generalized to the case of $r$-level UEP-LT as $\mathbb{E}\left[m_{o, i}^{j=1,(l+1)}\right]=$

$$
\sum_{d} \omega_{1, d} \varphi^{-1}\left(1-Z\left(1-\sum_{n} I_{1, n} \varphi\left((n-1) \mathbb{E}\left[m_{o, i}^{1,(l)}\right]\right)\right)^{d-1}\right)
$$

where, $\varphi(x)=1-\frac{1}{2 \sqrt{\pi x}} \int_{-\infty}^{\infty} \tanh (y / 2) e^{-\frac{\left.(y-x)^{2}\right)}{4 x}} d y$, and $\omega_{1, d}$ is the output edge degree coefficient for the first window. Moreover, $Z=\mathbb{E}[\tanh (z / 2)]$, where $z$ is the channel LLR, and in the case of BSC with bit cross-over probability $\epsilon$ is given by $z=\ln \left(\frac{1-\epsilon}{\epsilon}\right)$. This results in $Z=(2 \epsilon-1)^{2}$. At the first iteration, $\mathbb{E}\left[m_{o, i}^{j,(0)}\right]=0$, and for the sake of calculations, we use the approximation $\varphi(x) \approx$ $\exp \left(-0.4527 x^{0.86}+0.0218\right)[10]$.

\subsection{Design of Degree Distribution for UEP-LT Codes}

In [7], the authors explain that the Gaussian approximation of the messages sent from output to input nodes is not always true. In fact, asymptotically the messages sent from input nodes to output nodes are symmetrically normally distributed under the Central Limit theorem. Nevertheless, the messages passed from output nodes to input nodes are not Gaussian random variables because of irregular degrees of input nodes. Therefore, one can evaluate the mean of a message sent from an output node of degree $d_{j}$ to an input node of the $j^{\text {th }}$ level at the $l^{\text {th }}$ iteration of BP as

$$
\xi_{d_{j}}(\mu) \triangleq 2 \mathbb{E}\left[\operatorname{atanh}\left(\tanh (z / 2) \prod_{i=1}^{d_{j}-1} \tanh \left(U_{i} / 2\right)\right)\right]
$$

where $U_{i}$ 's $\left(i=1, \ldots, d_{j}-1\right)$ are iid symmetric Gaussian random variables with mean $\mu$ and variance $2 \mu$ representing messages $m_{i, o}^{j,(l)}$. Furthermore, the channel LLR is independent of the $U_{i}$ 's.

Now, if the objective is to keep working at a rate close to channel capacity, it is desired to minimize the number of encoded (output) nodes, or, to minimize the number of encoded bits generated by each level, that is to say, $N_{j}=p_{j} N$. It can be easily verified that

$$
N_{j}= \begin{cases}\alpha_{r} K_{r} / \beta_{r} & , j=r \\ \left(1-\frac{1}{\alpha_{j}} \sum_{i=j+1}^{r} \beta_{i} N_{i} / K_{i}\right) \frac{\alpha_{j} K_{j}}{\beta_{j}} & , 1 \leq j \leq r-1\end{cases}
$$

Therefore, starting from the last window backwards to the first, and plugging $\beta_{j}=1 /\left(\sum_{d_{j}=1}^{D_{j}} \omega_{j, d_{j}} / d_{j}\right)$ into (7), $N_{j}$ can be minimized with respect to the variables $\left\{\omega_{j, d_{j}}\right\}_{d_{j}=1}^{D_{j}}$. Here, $\omega_{j, d_{j}}$ and $D_{j}$ are the edge degree coefficients and the order of the degree distribution corresponding to the $j^{\text {th }}$ window, respectively.

The constraint of the optimization problem is that the mean of the messages passed from input to output nodes in each window increases after each iteration of the BP, i.e., $\mu^{j,(l+1)}>\mu^{j,(l)}$, or,

$$
\sum_{d_{j}=1}^{D_{j}} \omega_{j, d_{j}} \xi_{d_{j}}\left(\mu_{i}\right) \sum_{m=1}^{j} \alpha_{m} \frac{K_{m}-K_{m-1}}{K_{j}}>\mu_{i}, \forall i=0, \ldots, M-1
$$

where we discretize the continuous function $\xi_{d_{j}}(\mu)$ by having $M$ equidistance points. By this constraint, it is guaranteed that the bit error rate decreases at the next iteration. Thus, $r$ linear programs can be formulated with respect to the variables $\left\{\omega_{j, d_{j}}\right\}_{d_{j}=1}^{D_{j}}$ (or equivalently $\left\{\Omega_{j, d_{j}}\right\}_{d_{j}=1}^{D_{j}}$ ) which are solved from the last problem backwards up to the first one, recursively. Here, due to the lack of space, we only translate (7) $(1 \leq j \leq r-1)$ and (8) into the following optimization formulation

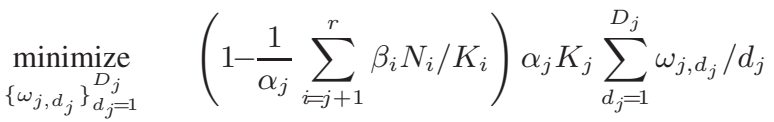

$$
\begin{aligned}
& \text { subject to } \quad \sum_{d_{j}=1}^{D_{j}} \omega_{j, d_{j}} \xi_{d_{j}}\left(\mu_{i}\right) \sum_{m=1}^{j} \alpha_{m} \frac{K_{m}-K_{m-1}}{K_{j}}>\mu_{i} \\
& \sum_{d_{j}=1}^{D_{j}} \omega_{j, d_{j}}=1, \omega_{j, d_{j}} \geq 0, \forall i=0, \ldots, M-1 .
\end{aligned}
$$

It is also note-worthy that the choices of $\mu_{0}, \alpha_{j}$ result in feasible or infeasible solutions. The value of $\mu_{0}$ should not be too large because it reduces the set of feasible solutions by increasing the set of inequality constraints.

\section{NUMERICAL RESULTS}

In this section, we numerically evaluate the performance of our scheme for some source-channel coding parameters, and we compare them with the existing results.

In the simulation, we assume that the source is uniformly distributed in the unitary interval, and the source coder is a binary truncated expansion, i.e., $\lambda=2$ in (3). For channel coding design, we first generate $\xi_{d}(\mu)\left(d=1, \ldots, D_{r}\right)$ using Monte-Carlo simulations, and then solve the optimization problem (9) for EEP-LT, 
2-level UEP-LT and 3-level UEP-LT codes using CVX, a package for specifying and solving convex programs [11]. Note that the EEP-LT design is performed by letting $p=0$ which boils down to the optimization problem in [7]. To be able to keep the problem convex we combine the linear programming with a grid search for values $0 \leq p \leq 1$ and $10 \leq \alpha \leq 50$ with step sizes $\Delta p=0.05$ and $\Delta \alpha=0.5$. For this purpose, we fix $\mu_{0}=16$, the step-size as $\Delta \mu=0.01$, and the degree orders as $D=90$ for EEP-LT, $D_{1}=60$ and $D_{2}=90$ for 2-level UEP-LT, and $D_{1}=60, D_{2}=70$ and $D_{3}=90$ for 3-level UEP-LT codes. The bit cross-over probability of the binary symmetric channel is $\epsilon=0.11$ for which the channel capacity is approximately 0.5 . The joint optimization yields $\alpha=16$ for EEP-LT codes, $(\alpha=20, p=0.65)$ for 2-level UEP-LT, and $\left(\alpha=28, p_{1}=0.6, p_{2}=0.3, p_{3}=0.1\right)$ for 3 -level UEP-LT codes.

The performance is evaluated by generating the codewords using the optimized degree distributions for the channel code rate $1 / 2$ (BSC with $\epsilon=0.11$ ). We consider the scenario in which the binary expansion truncates at $K=1000$ information bits. Furthermore, the length of the MIB window is fixed at $K_{1}=0.25 K$ for 2-level UEP-LT, and the corresponding windows for 3-level UEPLT codes are of length $K_{1}=0.2 K$ and $K_{2}=0.25 K$. The BP algorithm is also run for 60 iterations.

The simulation results (solid curves) and the asymptotic upper bounds (dashed curves), obtained from (6), by considering the optimized degree distributions, are illustrated in terms of MSE versus inverse channel code rate $R^{-1}$ in Figure 2 for EEP-LT and UEP-LT schemes. We also compare these results with the performance obtained by simulation of LT codes with unoptimized degree distributions in the tandem source-channel coding framework. In Figure 2, the dash-dotted curves show the performance of 2-UEP-LT and 3-UEP-LT codes with degree distributions studied in [5]. The 2-UEP-LT codes have the robust Soliton degree distribution[6] $\Omega_{1}\left(K_{1}=0.1 K, \delta=0.5, c=0.03\right), p_{1}=0.11$ for the first window, and Shokrollahi's degree distribution[8] for the second window. The 3-UEP-LT codes consist of the robust Soliton degree distribution $\Omega_{1}\left(K_{1}=0.1 K, \delta=0.2, c=0.03\right), p_{1}=0.084$, and the same distribution for the second window with parameters $\Omega_{2}\left(K_{1}=0.15 K, \delta=0.5, c=0.03\right), p_{2}=0.11$, and $\Omega_{3}(x)$ is the Shokrollhai's degree distribution. It is obvious that the optimized UEP-LT scheme outperforms the unoptimized ones in the sourcechannel coding scheme. Furthermore, the upper bounds, which approaches the simulation results at large block length, provide tight approximations of the MSE using optimized UEP-LT codes.

\section{CONCLUSION}

We study the performance of tandem source-channel coding by designing rateless channel coder in the high resolution quantization region. The aim of the paper is to optimize degree distributions for LT channel codes with unequal error protection property that minimize the end-to-end MSE of the source-channel coding system. The UEP-LT schemes with optimized degree distributions are obviously superior to the unoptimized UEP-LT schemes in the introduced framework. We also compare our results with the theoretical upper bounds on MSE for the proposed system which give tight approximations specially at large block length. Optimizing the windows' length of the UEP encoding scheme can be a subject of future work. Another future work is to employ the proposed method in order to design optimized degree distribution for anytime coding systems in which the performance of control applications in terms of end-to-end distortion and delay are taken into account as well as stability.

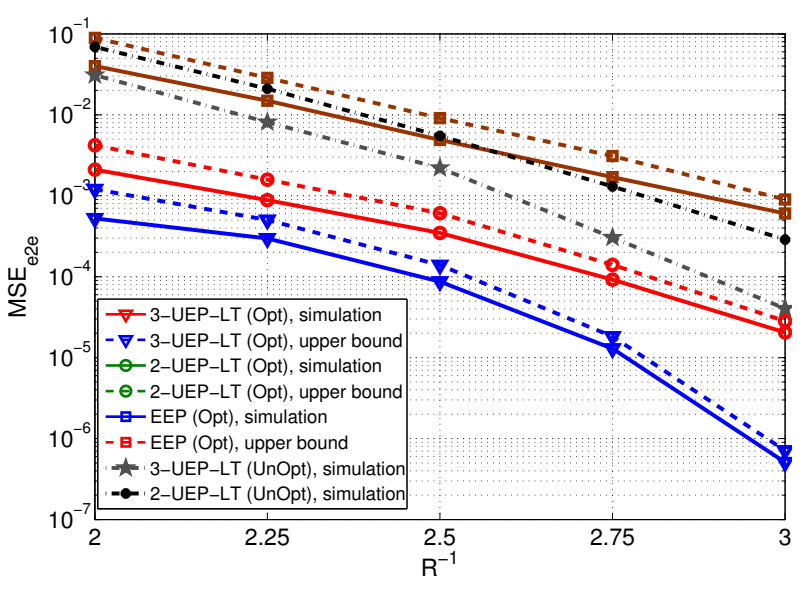

Figure 2: MSE comparison between UEP-LT and EEP-LT codes in the tandem source-channel coding.

\section{REFERENCES}

[1] R. G. Gallager, Information Theory and Reliable Communication. NY, USA: John Wiley \& Sons, Inc., 1968.

[2] G. Como, F. Fagnani, and S. Zampieri, "Anytime reliable transmission of real-valued information through digital noisy channels," vol. 48, no. 6, pp. 3903 -3924, April 2010.

[3] J. W. Modestino and D. G. Daut, "Combined source-channel coding of images," IEEE Trans. Commun., vol. 27, no. 11, pp. $1644-1659$, Nov. 1979.

[4] N. Rahnavard, B. Vellambi, and F. Fekri, "Rateless codes with unequal error protection property," IEEE Trans. Inf. Theory, vol. 53, no. 4, pp. 1521 -1532, April 2007.

[5] D. Sejdinovic, D. Vukobratovic, A. Doufexi, V. Senk, and R. Piechocki, "Expanding window Fountain codes for unequal error protection," IEEE Trans. Commun., vol. 57, no. 9, pp. $2510-2516$, Sep. 2009.

[6] M. Luby, "LT codes," IEEE Symposium on Foundations of Computer Science, pp. 271 - 280, 2002.

[7] O. Etesami and A. Shokrollahi, "Raptor codes on binary memoryless symmetric channels," IEEE Trans. Inf. Theory, vol. 52, no. 5, pp. 2033 - 2051, May 2006.

[8] A. Shokrollahi, "Raptor codes," IEEE Trans. Inf. Theory, vol. 52, no. 6, pp. $2551-2567$, June 2006.

[9] T. Richardson, M. Shokrollahi, and R. Urbanke, "Design of capacity-approaching irregular low-density parity-check codes," IEEE Trans. Inf. Theory, vol. 47, no. 2, pp. 619-637, Feb. 2001.

[10] S.-Y. Chung, T. Richardson, and R. Urbanke, "Analysis of sum-product decoding of low-density parity-check codes using a Gaussian approximation," IEEE Trans. Inf. Theory, vol. 47, no. 2, pp. 657 -670, Feb. 2001.

[11] M. Grant and S. Boyd, "CVX: Matlab software for disciplined convex programming, version 1.21," http://cvxr.com/cvx, Jul. 2010. 